(2) Open Access Full Text Article

CASE REPORT

\title{
Direct thrombolysis of multiple thrombi in both right and left heart atrium in a patient on extracorporeal membrane oxygenation support following urgent double-lung transplantation:
}

\section{a case report}

\author{
Lukas Pollert' \\ Zuzana Prikrylova' \\ Jan Berousek' \\ Frantisek Mosna' \\ Robert Lischke ${ }^{2}$ \\ 'Department of Anaesthesiology \\ and Intensive Care, 2nd Faculty of \\ Medicine, ${ }^{2} 3$ rd Surgical Department, \\ Ist Faculty of Medicine, Charles \\ University in Prague and Motol \\ University Hospital, Praha, Czech \\ Republic
}

\author{
This article was published in the following Dove Press journal: \\ Therapeutics and Clinical Risk Management \\ 16 June 2016 \\ Number of times this article has been viewed
}

\begin{abstract}
Background: Lung transplantation is considered an established treatment for patients with end-stage chronic respiratory failure. Patients with acute respiratory failure requiring respiratory support with invasive mechanical ventilation while awaiting lung transplantation are at high risk of death. Extracorporeal membrane oxygenation (ECMO) has been proposed as an alternative bridging strategy to mechanical ventilation. The shear stress created by the mechanical pumps causes changes in the hematological system in almost all patients treated with ECMO. An antithrombotic strategy to mitigate ECMO bleeding and thrombotic complications is necessary. The use of thrombolytic therapy is recommended for patients with acute symptomatic embolism with associated hypotension or shock. In this setting, the hemodynamic benefits of thrombolytic
\end{abstract} treatment far outweigh its bleeding risk.

Case presentation: This case report describes a 32-year-old woman suffering from lymphangioleiomyomatosis, who underwent urgent double-lung transplantation. This patient was maintained on ECMO preoperatively, perioperatively, and postoperatively due to life-threatening hypoxemia caused by the progression of her pulmonary tissue damage. Multiple thrombi developed in the early postoperative period, in both right and left heart atria. Direct thrombolysis was successfully performed on the first postoperative day.

Conclusion: According to the current published literature, direct thrombolysis of thrombi in both right and left atria in a patient supported on ECMO following urgent double-lung transplantation is an extremely rare treatment method. Even when taking into account all of the risks associated with thrombolysis and arteriovenous ECMO support, we found that this technique is very effective and, without a doubt, it saved the life of our patient.

Keywords: thrombolysis, double-lung transplantation, extracorporeal membrane oxygenation, general anesthesia, lymphangioleiomyomatosis

\section{Introduction}

Lung transplantation is considered an established treatment for patients with end-stage chronic respiratory failure. ${ }^{1}$ The disproportionately higher number of candidates in comparison to available organs explains the long waiting times and high risk of mortality while on the waiting list. ${ }^{1}$ The lack of efficacious and safe means of artificial respiratory support for patients who develop acute respiratory failure with refractory hypoxemia and hypercapnia contributes to the high mortality rate in patients

\section{Correspondence: Lukas Pollert} Intensive Care, 2nd Faculty of Medicine, Charles University in Prague and Motol University Hospital, V Úvalu 84, I50 06 Prague 5, Czech Republic

Tel +420 224435400

Fax +420224435420

Email lukas.pollert@fnmotol.cz 
awaiting transplantation. ${ }^{2}$ Extracorporeal membrane oxygenation (ECMO) has been proposed as an alternative bridging strategy to mechanical ventilation. In most studies, patients awaiting lung transplantation who were supported by ECMO were also mechanically ventilated. It is well known that high inspiratory pressures and high fractions of inspired oxygen are dangerous, and therefore, ECMO is an alternative, rather than an adjunct, to invasive mechanical ventilation and provides a better bridging strategy to lung transplantation. ECMO acts as a bridge for these patients and could provide an acceptable 1-year survival rate. ${ }^{3-5}$ The shear stress created by the mechanical pumps can cause changes in the hematological system, for example, acquired von Willebrand syndrome occurs in almost all patients treated with ECMO. An antithrombotic strategy to mitigate ECMO bleeding and thrombotic complications is necessary. ${ }^{6}$ Due to the high risk in all patients with the ECMO support, including the lung transplant patients for both bleeding and thrombotic complications, which can reach $5 \%$, experts in hemostasis can significantly affect the care of patients requiring mechanical circulatory support, and are a necessary part of the management team. ${ }^{6,7}$ Massive thrombosis in the early postoperative period can be a severe complication in transplant surgery. ${ }^{8}$ Catheter-related atrial thrombosis is a potentially deadly complication of intravascular devices. ${ }^{9}$ Hemodynamic status remains the most important short-term prognostic factor for patients with acute massive thrombosis.

Thrombotic complications involving the pulmonary veins and left atrium are rare after lung transplantation but in most cases end fatally after primary tissue rejection. A possible solution would be urgent reoperation, although the procedure would have an extremely high mortality. ${ }^{10}$ Our case report describes the thrombolysis of multiple thrombi in both right and left heart atrium, as a life-threatening complication in a patient with ECMO support following urgent bilateral lung transplantation. Written informed consent was obtained from the patient for publication of this case report and any accompanying images. Ethical approval for this case report was obtained from the Ethics Committee of the University Hospital Motol.

\section{Case presentation}

Our case report describes a 32-year-old woman diagnosed with lymphangioleiomyomatosis who was placed on an organ transplant waiting list in February 2014, due to rapidly progressing respiratory insufficiency. Her history was significant for multiple lymphangioleiomyomas in the retroperitonium along with repeated spontaneous pneumothoraces that required drainage. Transesophageal echocardiography (TEE) was performed during preoperative assessment and the patient was found to have a low ejection fraction. In March 2014, the patient was intubated and ventilated due to respiratory failure. She went on to develop bronchopneumonia caused by multiresistant Pseudomonas aeruginosa, followed by sepsis and disseminated intravascular coagulopathy with severe bleeding from the nose, hypopharynx, and lower respiratory tract. At this point, mechanical ventilation was no longer sufficient to sustain oxygenation and venovenous ECMO (VV-ECMO) was commenced, as a bridge to urgent lung transplantation (Figure 1). Six days later a donor was found and sequential bilateral lung transplant with a clamshell incision was performed with the support of the arteriovenous ECMO (AV-ECMO). The cannulae from the VV-ECMO were left in place and an arterial cannula was inserted centrally into the ascending aorta. Cannulation of the vena jugularis interna dextra was terminated. Due to anatomic disproportion, the donor lungs were partially resected at the right middle lobe and lingula.

Perioperative TEE showed severely hypokinetic right and left ventricles along with a large thrombus in the left atrium near the pulmonary veins (Figure 2). The procedure was complicated by diffuse bleeding due to coagulopathy present in the septic state, which resulted in a $10 \mathrm{~L}$ blood loss. Postoperatively, the AV-ECMO was left in place due to the poor myocardial function; therefore, the sternotomy was not closed to allow the arterial cannula to remain in the aorta. During admission to the intensive care unit, severe pulmonary edema was noted with the continuous leakage of edematous fluid from the endotracheal tube. Tidal volumes

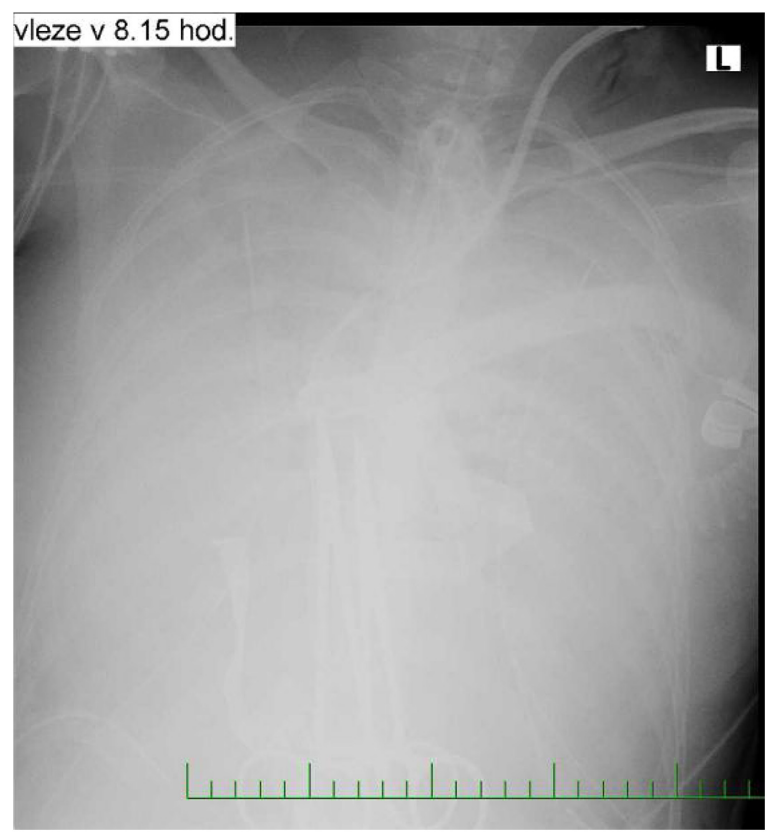

Figure I Chest $X$-rays on venovenous extracorporeal membrane oxygenation (VV-ECMO) before thrombolysis. 


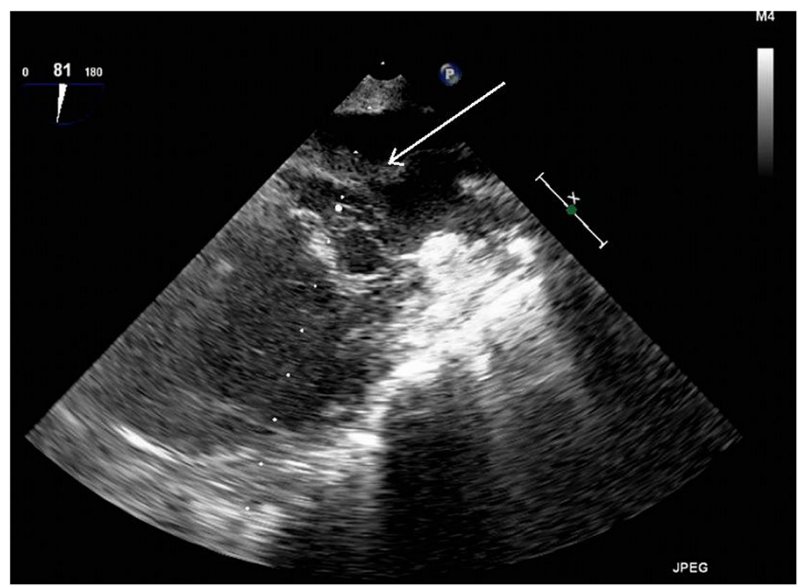

Figure 2 Transesophageal echocardiography (TEE) view with thrombus in left atrium before thrombolysis.

were $30-40 \mathrm{~mL}$ with a peak pressure of $30 \mathrm{~cm} \mathrm{H}_{2} \mathrm{O}$ and a positive end-expiratory pressure of $10 \mathrm{~cm} \mathrm{H}_{2} \mathrm{O}$. Cardiovascular support with high-dose norepinephrine $(>1 \mu \mathrm{g} / \mathrm{kg} / \mathrm{min})$ was in place. Arterial blood gas results showed good oxygenation due to the support of AV-ECMO. Immunosuppression therapy was commenced with tacrolimus, mycophenolate mofetil, and corticosteroids.

Anticoagulation with heparin was initiated with a target of activated coagulation time (ACT) 150-200 seconds. Six hours later, a TEE was performed and thrombi were noted in both the left and right atria. Due to the high risk of embolism, we were faced with making a crucial decision: to start thrombolysis, which is generally contraindicated in immediate postoperative period, or not. We evaluated that the risk of massive embolism was superior to the risk of bleeding when the sternotomy was not closed. The primary thrombolysis was undertaken with $25 \mathrm{mg}$ alteplase given over 2 hours into the pulmonary artery as the rescue procedure declared in medical documentation. The left atrial thrombus was quickly reduced, after which a second identical dose of alteplase was given. Pulmonary edema began to resolve after the completion of thrombolysis. A further TEE showed no thrombi in the left ventricle or pulmonary veins, although small thrombi remained in the right and left atria. The following day a full dose of thrombolytic agent was given - $50 \mathrm{mg}$ alteplase over 2 hours intravenously - after which no further thrombi could be detected on TEE (Figure 3). Lung function continued to improve along with radiological findings within 1 week following the thrombolysis (Figure 4).

In the following days, the patient developed acute kidney injury for which she was placed on continuous renal replacement therapy. Gradually, her heart and lung function improved and on postoperative day 8, ECMO support was stopped. The

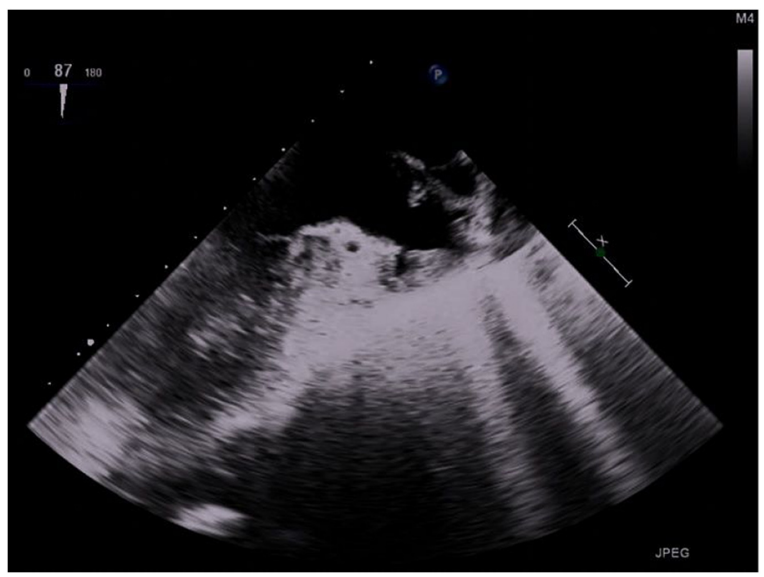

Figure 3 Transesophageal echocardiography (TEE) view with no thrombus in left atrium after thrombolysis.

sternotomy was closed after resolution of the $P$. aeruginosa infection. Weaning and rehabilitation were complicated by poor muscle strength. Due to worsening lung function, a transbronchial biopsy was performed which confirmed tissue rejection A 1-2 (histologic grading of lung allograft rejection- the Classification of Pulmonary Allograft Rejection: Lung Rejection Study Group) and was successfully treated with pulses of steroids. The following days were significant for the loss of consciousness that was thought to be caused by posterior reversible encephalopathy syndrome. An electroencephalograph showed an epileptic status, and antiepileptic medications were prescribed. Three months postoperatively, stenosis of the right main bronchus was diagnosed bronchoscopically, and a biodegradable stent ELLA $^{\circledR}$ (ELLA-CS s.r.o. Milady Horakove, Hradec Kralove, Czech Republic) was implanted. Another setback of pulmogenic sepsis and multiorgan failure, with a need for continuous renal replacement therapy occurred. Six months postoperatively, the patient was extubated and began to actively rehabilitate. Seven months postoperatively, the patient was discharged to home.

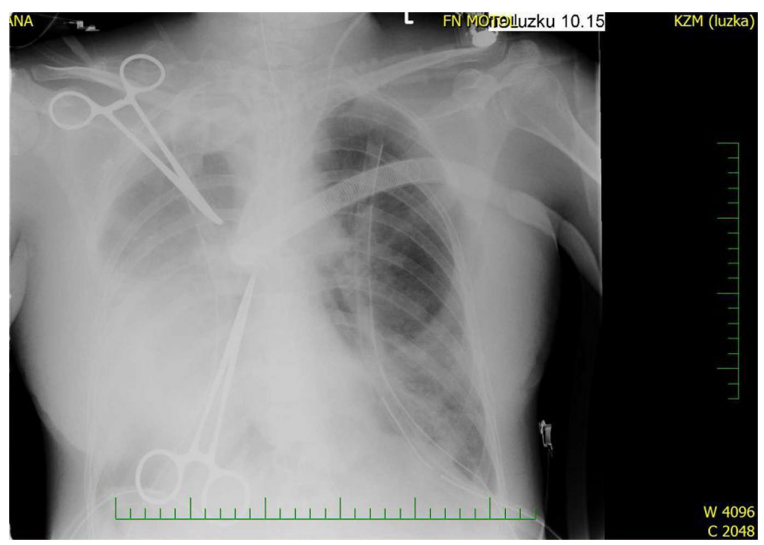

Figure 4 Chest X-rays, I week after thrombolysis. 


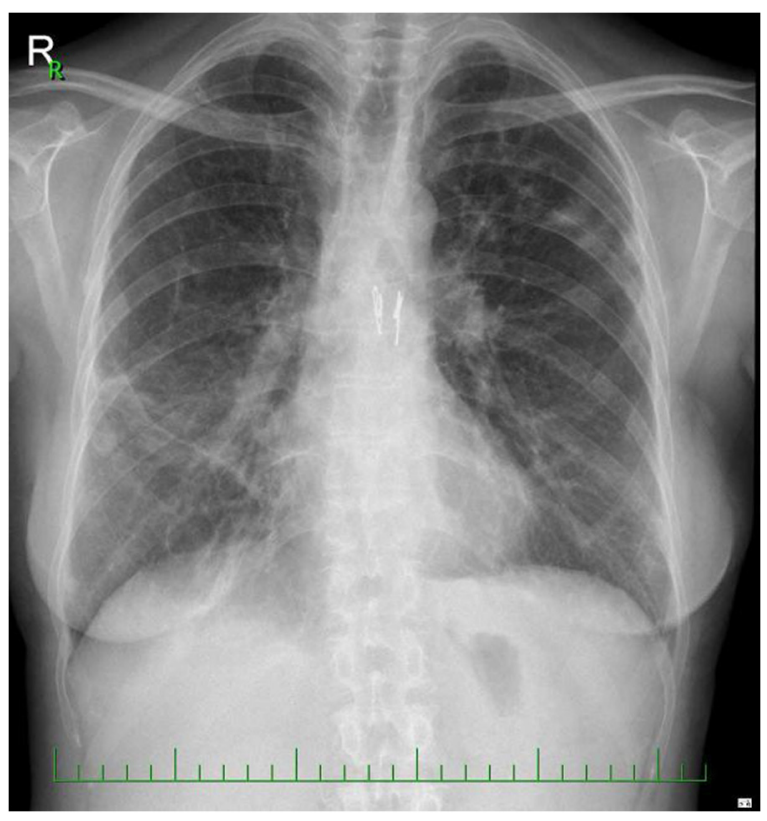

Figure 5 Chest $X$-rays, I year following DLTx. Abbreviation: DLTx, double-lung transplantation.

Currently, she enjoys a good quality of life without any signs of stenosis of the upper respiratory tract and with stationary spirometric values (last forced expiratory volume in 1 second $\left[\mathrm{FEV}_{1}\right] 38 \%$ ) (Figure 5).

\section{Discussion}

We showed that early ECMO support in patients with critical hypoxemia is advantageous. ECMO is a lifesaving option for patients with interstitial lung disease and acute respiratory failure provided they are candidates for lung transplantation. . $^{3,41}$ ECMO is not able to reverse the poor prognosis in patients who do not qualify for lung transplantation. ${ }^{11,12}$ Chiumello et al referred, that the report by the United Network of Organ Sharing showed that, despite its complexity and side effects, the use of ECMO as a bridge to lung transplantation has risen by $150 \%$ in the last 2 years compared to the previous decades (1970-2010). ${ }^{3}$ ECMO is an alternative, rather than an adjunct, to invasive mechanical ventilation and is a better bridging strategy to lung transplantation.,11,12 Extracorporeal life support as a bridge to lung transplantation is a promising option for patients with end-stage lung disease on the organ transplant waiting list. ${ }^{13}$ The shear stress created by the mechanical pumps causes changes in the hemocoagulation system in almost all patients treated with ECMO ${ }^{9}$ ECMO and the surgical procedure carry a risk of considerable bleeding which is caused by the surgical wound itself and by the use of heparin along with other causes such as hypothermia, anemia, inflammation, and/or drug effects (eg, antiplatelet agents, low-molecular-weight heparins). ${ }^{13}$ Massive bleeding leads to massive allogeneic blood transfusions, which are a known independent risk factor for poor wound healing. ${ }^{14}$ ECMO carries a high risk for both bleeding and thrombotic complications. ${ }^{6,7}$ Massive thrombosis in the early postoperative period can be a severe complication in transplant surgery. ${ }^{8}$ Catheter-related atrial thrombosis is a potentially deadly complication of intravascular devices. ${ }^{9}$ Hemodynamic status remains the most important short-term prognostic factor for patients with acute massive thrombosis. ${ }^{6}$ In concordance with the use of thrombolytic therapy recommendation for patients with acute symptomatic pulmonary embolism, we decided to thrombolyse with tissue plasminogen activator. ${ }^{8,15,16}$ In this setting, the hemodynamic benefits of thrombolytic treatment far outweigh its risk for bleeding. ${ }^{10}$ The left atrial cuff technique was successfully adopted for clinical lung transplantation. Thrombosis of the left atrium and pulmonary veins is a rare but very serious complication of lung transplantation and carries with it a high mortality and morbidity, and therefore attention to details when performing the left atrial anastomosis will minimize this complication. ${ }^{17}$ The diagnosis of pulmonary vein obstruction after lung transplantation is difficult because other conditions, such as primary graft failure or posttransplantation reperfusion edema, may show similar radiographic changes. ${ }^{16}$ Clinical findings of diffuse interstitial edema, consolidation, excessive serous bronchorrhea, pleural tube drainage, or elevated pulmonary wedge pressure in the transplanted lung are suggestive of pulmonary venous obstruction. ${ }^{16}$ Pulmonary angiogram shows a significant delay in the transit of contrast into the affected side. However, the detection of a thrombus in the left atrium by angiography is difficult. ${ }^{8}$ TEE has been shown to be a superior technique for visualization of the left atrium and pulmonary veins; its role in the diagnosis of left atrial thrombus is well documented. ${ }^{8}$ This also underlines the importance of perioperative TEE during lung transplantation. ${ }^{3,4}$ When the diagnosis of left atrial thrombus and pulmonary venous obstruction after lung transplantation is made, one treatment option is surgical correction which should be undertaken as soon as possible to salvage the transplanted lung and the patient's life. ${ }^{17}$ Care must be taken not to dislodge the left atrial thrombus and thus cause arterial thromboembolism. As regards our patient's case, thrombi were noted in both left and right atria and she had severe coagulopathy. Therefore, surgery was contraindicated and we decided to administer primary thrombolytic agent despite the bleeding risk. The results proved excellent.

Alteplase is a fibrinolytic agent that is indicated for the treatment of massive embolism in intermediate- and high-risk patients. ${ }^{8,18}$ Controversy exists as to the patient population that will benefit most from fibrinolytic therapy, as well as the 
proper dose and administration technique. The patient's risk of bleeding should be weighed against the potential benefits of treatment in light of the clinical presentation because of the high mortality rate associated with pulmonary embolism. ${ }^{15}$ Due to the high risk of bleeding within the first postoperative day, we decided to administer a half dose of the alteplase following other authors' suggestions. ${ }^{10,18}$ To minimalize the risk of bleeding, we checked the hemocoagulation using viscoelastometric assays. ${ }^{7}$ Following other authors' recommendations, we followed a restrictive blood transfusion strategy. ${ }^{19}$ The fibrinogen level $<1.5 \mathrm{~g} / \mathrm{L}$ during thrombolysis was not associated with an increased bleeding risk. ${ }^{16,20}$ It is well documented that larger doses as well as increased duration of thrombolysis are associated with an increased risk of bleeding. ${ }^{20}$ Surgical thrombectomy is an alternative method to thrombolysis; however, it carries an increased risk for critically ill patients. ${ }^{21}$ Postoperative TEE enabled early diagnosis of massive thrombi, which developed as a complication of the ECMO support despite the administration of unfractionated heparin which maintained the ACT between 180 and 220 seconds. ${ }^{8,22}$ It is proven that in patients with shorter ACT values or those supported on ECMO with no anticoagulation, there are higher number of thrombotic or thromboembolic complications than in patients with severe trauma or massive traumatic bleeding. . $^{2324}$

\section{Conclusion}

Massive thrombus formation in the left atrium is a lifethreatening situation. Unfortunately, the treatment of choice, surgery, was contraindicated in our patient. ECMO bridging to lung transplantation is considered to be a safe method with good long-term outcome. Pharmacological thrombolysis is the method of choice for the treatment of thrombotic complications of ECMO following lung transplantation. Our case report explores a patient with severe bleeding due to sepsis and coagulopathy, an open sternotomy, and connection to AV-ECMO with a central cannula and who underwent the successful administration of thrombolytic agent 2 hours post-lung transplantation. It is important to continue to develop and enhance the level of knowledge regarding these critical clinical situations, which is currently lacking in published literature.

\section{Author contributions}

ZP has made substantial contributions to conception and design, acquisition, analyzing, and interpretation of data and has been involved in drafting the manuscript or revising it critically for important intellectual content. FM has made substantial contributions to conception and design, acquisition, analyzing, and interpretation of data. JB has made substantial contributions to conception and design, analysis, and interpretation of data and has been involved in drafting the manuscript and revising it critically for important intellectual content and in giving final approval of the version to be published. LP made substantial contributions to conception and design, analysis, and interpretation of data and has been involved in drafting the manuscript and revising it critically for important intellectual content and in giving final approval of the version to be published. ZP, JB, and LP agreed to be accountable for all aspects of the work in ensuring that questions related to the accuracy or integrity of any part of the work are appropriately investigated and resolved. RL has made contributions to acquisition and interpretation of data and has critically revised the manuscript for important intellectual content. All authors contributed toward data analysis, drafting and critically revising the paper and agree to be accountable for all aspects of the work.

\section{Disclosure}

The authors report no conflicts of interest in this work.

\section{References}

1. Myles PS, Snell GI, Westall GP. Lung transplantation. Curr Opin Anaesthesiol. 2007;20:21-26.

2. Diaz-Guzman E, Hoopes CW, Zwischenberger JB. The evolution of extracorporeal life support as a bridge to lung transplantation. ASAIOJ. 2013;59:3-10

3. Chiumello D, Coppola S, Froio S, Kolombo A, Del Sorbo L. Extracorporeal life support as bridge to lung transplantation: a systematic review. Crit Care. 2015;19:19.

4. Vymazal T, Krecmerova M. Respiratory strategies and airway management in patients with pulmonary alveolar proteinosis: a review. BioMed Res Int. 2015;1-5.

5. Inci I, Klinzing S, Schneiter D, et al. Outcome of extracorporeal membrane oxygenation as a bridge to lung transplantation: an institutional experience and literature review. Transplantation. 2015;99(8): $1667-1671$.

6. Baumann Kreuziger L, Massicotte MP. Mechanical circulatory support: balancing bleeding and clotting in high-risk patients. Hematology Am Soc Hematol Educ Program. 2015;2015(1):61-68.

7. Durila M, Lukáš $P$, Astraverkhava M, Vymazal T. Evaluation of fibrinogen concentrates and prothrombin complex concentrates on coagulation changes in a hypothermic in vitro model using thromboelastometry and thromboelastography. Scand J Clin Lab Invest. 2015;75: 407-414.

8. Reddick M, Pillai AK, Bollineni S, Kalva S. Catheter directed pharmacologic thrombolysis for acute pulmonary embolism immediately following lung transplantation. J Vasc Interv Radiol. 2015;26(1): 142-144.

9. Orgeron GM, Pollard JL, Pourmalek P, Sloane PJ. Catheter-directed low-dose tissue plasminogen activator for treatment of right atrial thrombus caused by a central venous catheter. Pharmacotherapy. 2015; 35(10):e153-e158.

10. Marshall PS, Tapson V, Jiménez D. Controversies in the management of life-threatening pulmonary embolism. Semin Respir Crit Care Med. 2015;36(6):835-841.

11. Przybysz TM, Heffner AC. Early treatment of severe acute respiratory distress syndrome. Emerg Med Clin North Am. 2016;34(1):1-14. 
12. Krecmerova M, Mosna F, Bicek V, et al. Whole lung lavage with the support of extra-corporal membrane oxygenation in patient with pulmonary alveolar proteinosis. BMC Anesthesiol. 2015;15:173.

13. Vymazal T, Filaun M, Horáček M. Impact of retransfusion of blood processed in cell-saver on coagulation versus cardiopulmonary bypass: a prospective observational study using thromboelastography. Biomed Pap Med Fac Univ Palacky Olomouc Czech Repub. 2015;159(1): 131-134.

14. Vymazal T, Horáček M, Duřpekt R, Hladíková M, Cvachovec K. Is allogeneic blood transfusion a risk factor for sternal dehiscence in cardiac surgery - a prospective observational study. Int Heart J. 2009; 50:601-608

15. Patel N, Patel NJ, Agnihotri K, et al. Utilization of catheter-directed thrombolysis in pulmonary embolism and outcome difference between systemic thrombolysis and catheter-directed thrombolysis. Catheter Cardiovasc Interv. 2015;86(7):1219-1227.

16. Peters NA, Paciullo CA. Alteplase for the treatment of pulmonary embolism: a review. Adv Emerg Nurs J. 2015;37(4):258-272.

17. Pham SM, Armitage JM, Katz WE, Griffith BP. Left atrial thrombus after lung transplantation. Ann Thorac Surg. 1995;59(2):513-515.
18. Martin L, Winter J, Carter K, Foertsch M, Takieddine S, Harger N. Standard versus low dose alteplase for treatment of acute pulmonary embolism. Crit Care Med. 2015;43(12 Suppl 1):21.

19. Vymazal T. Massive hemorrhage management - a best evidence topic report. Ther Clin Risk Manag. 2015;11:1107-1111.

20. Lee K, Istl A, Dubois L, et al. Fibrinogen level and bleeding risk during catheter-directed thrombolysis using tissue plasminogen activator. Vasc Endovascular Surg. 2015;49(7):175-179.

21. Kalem V, Buchwald D, Strauch J, et al. Surgical extraction after thrombosis around the Avalon dual lumen cannula. Ann R Coll Surg Engl. 2014; 96:106E-108E.

22. Viesselmann C, Rose A. Standardization of anticoagulation management in extracorporeal membrane oxygenation. Crit Care Med. 2015; 43(12 Suppl 1):227.

23. Arlt M, Philipp A, Voelkel S, et al. Extracorporeal membrane oxygenation in severe trauma patient with bleeding shock. Resuscitation. 2010; 81:804-809.

24. Haneke F, Schildhauer TA, Schlebes AD, Strauch JT, Swol J. Infections and extracorporeal membrane oxygenation: incidence, therapy and outcome. ASAIO J. 2015;62(1):80-86.
Therapeutics and Clinical Risk Management

\section{Publish your work in this journal}

Therapeutics and Clinical Risk Management is an international, peerreviewed journal of clinical therapeutics and risk management, focusing on concise rapid reporting of clinical studies in all therapeutic areas, outcomes, safety, and programs for the effective, safe, and sustained use of medicines. This journal is indexed on PubMed Central, CAS,

\section{Dovepress}

EMBase, Scopus and the Elsevier Bibliographic databases. The manuscript management system is completely online and includes a very quick and fair peer-review system, which is all easy to use. Visit $\mathrm{http}: / / \mathrm{www}$.dovepress.com/testimonials.php to read real quotes from published authors. 